\title{
Delivering Context-aware Learning Guidance in the Mobile Learning Environment based on Information Theory
}

\author{
Rita Kuo ${ }^{1}$, Min-Chang $\mathrm{Wu}^{2}$, Alex Chang ${ }^{2}$, Maiga $\mathrm{Chang}^{3}$ and Jia-Sheng $\mathrm{Heh}^{2}$ \\ ${ }^{1}$ Dept. of Electronic Engineer, Chung-Yuan Christian Univ. \\ ${ }^{2}$ Dept. of Information and Computer Engineering, Chung-Yuan Christian Univ. \\ ${ }^{3}$ Office of National Science and Technology Program for e-Learning in Taiwan \\ rita@mcsl.ice.cycu.edu.tw, alix@mcsl.ice.cycu.edu.tw, aslada@mcsl.ice.cycu.edu.tw, \\ maiga@ms2.hinet.net,jsheh@ice.cycu.edu.tw
}

\begin{abstract}
Mobile Learning makes students get the advantages of both traditional learning and e-learning. How to give students learning sequence suggestions in the mobile learning environment is a big issue and is also the goal of this paper. This research uses knowledge map to store the characteristics of each learning object and designs a situated map to represent spatial knowledge in the mobile learning environment. By using these two knowledge structures, knowledge map and situated map, the system created by this research can generate various navigation sentences and ask students to observe the characteristics of learning objects. This research also takes information theory into consideration in order to decide which navigation sentence should deliver to the student first. The system calculates the entropy for each generated sentence and finds the most appropriate one to deliver to the student. At the end of this paper, an experiment system is implemented for the 5th year biology course, Plant Observation.
\end{abstract}

Keywords: Mobile Learning, Learning Navigation, Situated Learning, Knowledge Map

\section{Introduction}

Mobile learning becomes an interesting issue in e-Learning field in recent years. Mobile learning is used for supporting students learning without time limit [6]. Students could connect to e-learning repository via mobile devices such as Personal Digital Assistant (PDA), mobile phone, tablet PC, and notebook, anytime and anywhere [10].

More and more schools improve the equipments of mobile device in order to apply mobile learning into teaching, meanwhile, researchers have proposed some challenge issues [6]. The goal of paper focuses on how to navigate students in the mobile learning environment according to what this environment has. In most of mobile learning researches, students still need teacher's assistances to guide them where to go and what to learn [2][3]. This research develops a system in order to generate guidance message for students when they are walking in a mobile learning environment with their mobile devices.

Section 2 introduces some related research backgrounds, including situated learning, spatial knowledge, and knowledge map. A situated map represented the spatial knowledge of the mobile learning environment is introduced in Section 3, moreover, the knowledge representation and the navigation generating process is also analyzed in this section. Section 4 uses information theory to decide navigation priority. A mobile learning navigating system is implemented and used to demonstrate the research results in Section 5. Section 6 gives a brief conclusion and some possible future works.

\section{Research Background}

Situated learning takes learning as a situated activity and believes that people learn new skills by practicing [9]. Situated learning theory is based on Vygotsky's researches which proposed that human could only develop themselves by social interactions [13]. Lave (1991) believes that human's everyday cognitions are related to their social situations and these cognitions construct their own knowledge representation in their minds. Furthermore, situated learning can make students construct more complete knowledge and allow them being able to apply what they have learned in solving real-life problems [1].

Applying situated learning into mobile learning environment could be an interesting issue, because the environment provides students a chance to 'touch' and 'observe' real learning objects. This research takes spatial knowledge proposed by Glasgow and Papadias in 1992 
when analyzes the navigation guidance [7]. Spatial knowledge only stores object locations without other information such as shape, size, color, etc. Furthermore, spatial knowledge also records the relations among different object locations [5]. The relations are categorized into various types, including direction relations (e.g., east, north-east), topological relations (e.g., inside, overlap), ordinal relations (a subset of topological relations), and distance relations (e.g., near, far) [11].

The navigation in mobile learning environment should not only focus on the location where students should go, but also the learning object's characteristics which students should are observed. For this reason, knowledge base would be an important component in designing navigation system in the mobile learning environment. This research uses Knowledge Map as the knowledge base. The knowledge architecture of the Knowledge Map involves two parts, Concept Hierarchy and Concept Schema, as Figure 1 shows [8].

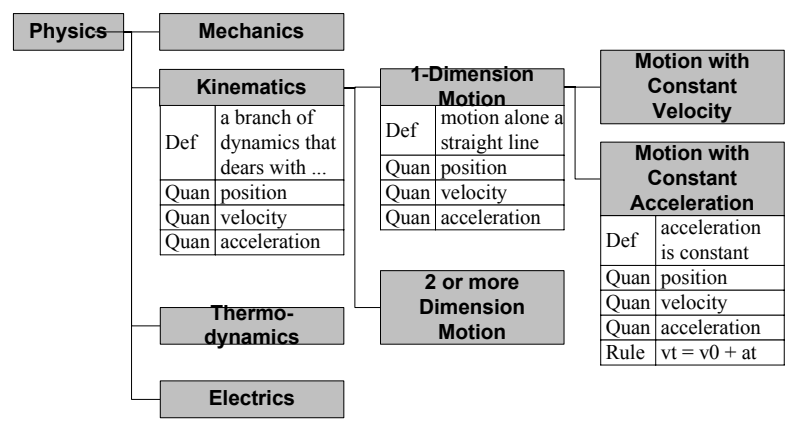

Figure 1. Example of Knowledge Map

1. Concept Hierarchy: concept hierarchy comes from the hierarchical network model [4]. Collins and Quillian have proved that human knowledge is stored in hierarchical architecture. Concept hierarchy presents the hierarchical relationships among concepts in the same domain. Different to the six links proposed by Dansereau, the relationships among concepts in concept hierarchy are only "part of $\left(r_{\text {part-of }}\right)$ " and "type of $\left(r_{\text {type-of } f}\right)$."

2. Concept Schema: schema is the generic concept data structure in memory [12]. Concept schema stores the concept attributes (denoted as $a_{m}$ ), such like the definitions and examples. Each concept $\left(c_{i}\right)$ in the concept hierarchy has links $\left(l_{c_{i}, a_{m}}\right)$ with its attributes $\left(a_{m}\right)$ in the concept schema. The link relation is called the relation schema $\left(r_{\text {schema }}\right)$.

This research integrates spatial knowledge and knowledge map into situated learning and tries to arrange proper learning path in the mobile learning environment. In order to achieve the goal, two major issues have to solve first. The first issue is how to store spatial knowledge and its related learning objects; and the second issue is how to decide where the student should go first in the mobile learning environment.

\section{Navigating Sentence Generation}

For storing spatial knowledge and related learning objects, this research defines Situated Map to record all learning locations in the mobile learning environment. Besides, the situated map also gathers the information about what learning objects existed in different learning locations. Based on its functionalities, there are three major elements in the situated map:

1. Area, $a_{u}$ : indicates learning locations.

2. Path, $p_{u, v}$ : presents the route between two areas.

3. Learning Object set, $O_{u}$ : denotes possible learning objects for learning area $a_{u}$.

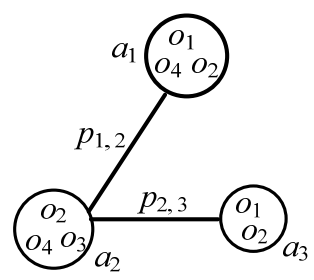

Figure 2. Example of Situated Map.

Figure 2 is an example of a situated map with three different learning locations. In area $a_{1}$, students could find three learning objects, $O_{1}=\left\{o_{1}, o_{2}, o_{4}\right\}$. Students could also go to area $a_{2}$ through the path $p_{1,2}$ and observe other learning objects not in $a_{1}$, such as learning object $o_{3}$. There is no path between $a_{1}$ and $a_{3}$. If students want to go to $a_{3}$ from $a_{1}$, they should go to $a_{2}$ first then go to $a_{3}$.

The details of learning objects are stored in knowledge map, which is mentioned in the previous section. Most of mobile learning activities ask students to verify various characteristics of learning objects. For this reason, the concepts stored in knowledge map can be simplified into three classes, the characteristic name $\left(c^{\text {name }}\right)$ represented by double border box, characteristic type of learning object $\left(c^{\text {type }}\right)$ represented by grey box, and learning object $\left(o_{i}\right)$ represented by white box.

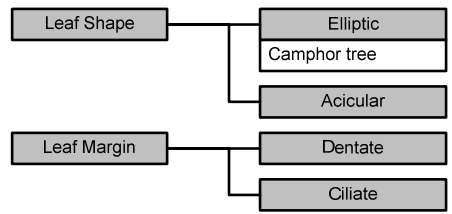

Figure 3. Example of Knowledge Map in Plant Observation

Take Figure 3 for example, "leaf shape" and "leaf margin" are the observable characteristics of plants and would be the parent levels of concept hierarchy; "Elliptic" and "Acicular" are the characteristic types of "leaf shape" 
and become its child nodes. Since the leaf shape of camphor tree is elliptic, the learning object of "elliptic" will be "camphor tree."

The system can use sentence template to create human readable sentence to generate navigating sentences. For example, if the system wants to generate a sentence in order to ask students paying attention on a learning object and its related characteristics, the sentence could be:

Please take a look at (learning object), its (characteristic name) is (characteristic type).

Using the related concepts and schemas in Figure 3, the sentence would be generated as

Please take a look at camphor tree, its leaf shape is elliptic.

Another sentence type, the interrogative sentence, could be also generated by using sentence template, such as

Please write down what type of (characteristic name) (learning object) is.

The system can construct a question as

Please write down what type of leaf shape camphor tree is.

With the knowledge map and sentence templates, the navigating system can generate various types of navigating sentences easily.

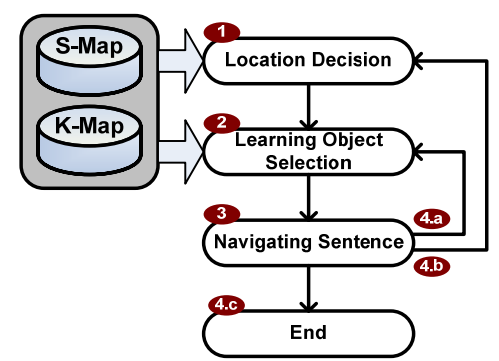

Figure 4. The flow of navigation strategy

Based on the previous analysis, Figure 4 draws the flowchart of navigation students in the mobile learning environment. The first two steps, step 1 and step 2, are locating the suitable learning spots and related learning objects from situated map and knowledge map. After that, the navigating system generates human readable sentences for students in step 3 . If there is no further learning objects needed to learn, the navigating system goes to the end step, step 4.c; if there is any other learning object that is required for learning in the same learning spot, the system goes back to step 2; and, if there is any other learning object that is required for learning but locates in the other spots, the system goes to step 1 .

\section{Entropy Calculation}

Information theory is used to decide where students should go and what they should observe as the step 1 and 2 showing in Figure 4. There are three measures can be used in deciding navigating objective:

(a) Learning Object Information: $I\left(o_{\mathrm{i}}\right)$

(b) Learning Area Information: $I\left(a_{u}\right)$

(c) Characteristic Information: $I\left(c_{\mathrm{j}}^{\text {type }}\right)$

\section{(a) Learning Object Information}

The calculation of learning object information is related the probability of selecting the learning object out in the mobile learning environment. The equation would be

$$
I\left(o_{i}\right)=\log _{2}\left(1 / p_{O_{i}}\right)
$$

Take learning object $o_{1}$ in Figure 2 for example. There are eight learning objects separates in the learning map and two of them are $o_{1}$. The probability to select $o_{1}$ for students is $2 / 8$. Based on the equation, the information of $o_{1}$ is

$$
I\left(O_{1}\right)=\log _{2}\left(\frac{1}{2 / 8}\right)=2
$$

All the information of learning objects could be calculated and represented as a vector $I(O)$ :

$$
I(O)=[2,1.41,3,2]
$$

\section{(b) Learning Area Information}

After the system has each learning object's information, each learning spot's information can be calculated consequently. The information of learning area is the total information from all learning objects exist in it.

$$
I\left(a_{u}\right)=\sum_{o_{i} \in a_{u}} I\left(o_{i}\right)
$$

In Figure 2, each learning spot's information is

$$
\begin{aligned}
& I\left(a_{1}\right)=2+1.41+2=5.41 \\
& I\left(a_{2}\right)=1.41+3+2=6.41 \\
& I\left(a_{3}\right)=2+1.41=3.41 .
\end{aligned}
$$

Therefore, the learning spot $a_{2}$ is the first location that the system will recommend students to go. 


\section{(c) Characteristics Information}

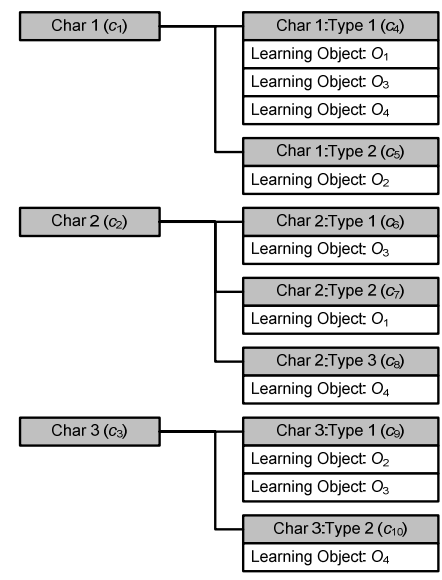

Figure 5. An Example of Knowledge for Mobile Learning Navigating System

When the system generates the navigating sentence will never use only the characteristic name. Because each characteristic type belongs to a characteristic name just like Figure 5 shows. The information of learning objects' characteristics are only related to the probability of selecting the characteristic types. For example, in Figure 5, the probability of selecting $c_{4}$ out is $(1 / 3)^{*}(1 / 2)=(1 / 6)$. Therefore, the information of characteristic $c_{4}$ is

$$
I\left(c_{4}\right)=\log _{2}\left(\frac{1}{1 / 6}\right)=2.585 \text {. }
$$

The vector $I\left(C^{\text {type }}\right)$ stores the information for all characteristics in concept hierarchy, and the $I\left(C^{\mathrm{type}}\right)$ for Figure 5 is

$$
I\left(C^{\text {type }}\right)=[2.585,2.585,3.170,3.170,3.170,2.585,2.585] .
$$

\section{Choosing Navigating Objective}

The process of choosing what navigating objective the system should pick up to generate the navigating sentence involves six steps. For example, if the system picks the Elliptic characteristic up from Figure 3, then the system can generate a navigating sentence:

"Please take a look at camphor tree, its leaf shape is elliptic."

The six steps of choosing navigating objective are:

1. choosing the learning spot according to the learning area information;

2. finding out what learning objects the chosen learning spot has;

3. finding out what characteristic types those learning objects have involved in;

4. picking the characteristic type with maximum information up;
5. if there are more than one learning objects involved in the characteristic type, then go to step 6 ; else the system takes the characteristic type as objective to generate the navigating sentence;

6. picking the learning object with maximum information up, the system will then use the characteristic type and the learning object to generate the navigating sentence.

Taking Figure 2 and Figure 5 for example, the first step chooses learning spot 2 for navigating; the second step finds there are learning object $o_{2}, o_{3}$, and $o_{4}$, in the spot 2; the third step finds that the learning object, $o_{2}, o_{3}$, and $o_{4}$, are involved in the characteristic type $c_{4}, c_{5}, c_{6}, c_{8}$, $c_{9}$, and $c_{10}$; the fourth step finds that the characteristic type $c_{6}$ and $c_{8}$ have the maximum information, 3.170; the fifth step finds that there are learning object $o_{3}$ and $o_{4}$ have involved in the characteristic types with maximum information; the sixth step picks learning object $o_{3}$ and the characteristic type $c_{6}$ as the objective to generate the navigating sentence.

\section{Example of Plant Observation}

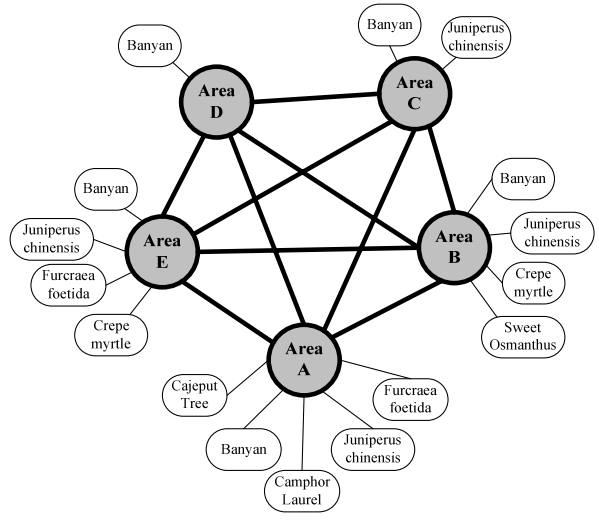

Figure 6. Situated Map in Plant Observation Lesson.

An experiment system is developed for the 5 th year biology course, Plant Observation, in Cherng-Gong Elementary School, Taoyuan, Taiwan. All the characteristics that students need to know are stored in the knowledge map. The teacher builds some instructional webpages to introduce each plant. The situated map has five near-by small gardens located in the campus. Figure 6 illustrates these five areas and their related learning objects.

Figure 7 shows the snapshot of the navigating system. The upper frame shows the instructional webpages. On the bottom left, a small map shows the learning area map. The navigating sentence is displayed on the bottom right frame. Students can write down the answer in the text field and send the answer back to the server. 


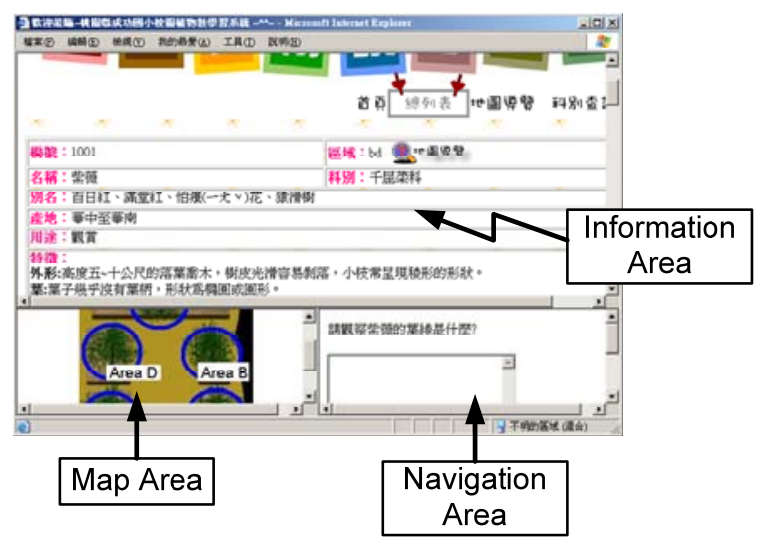

Figure 7. A snapshot of navigating system

\section{Conclusion}

This paper shows a way to generate the navigating sentence to recommend students where to go and what to learn in the mobile learning environment according to what learning objects the environment has. To realize this idea, this research develops the situated map to represent the spatial knowledge in the learning environment. The related characteristics of learning objects are stored in knowledge map. Using these two knowledge structures, the system can generate various types of navigating sentences.

There are still some issues could be discussed to improve the navigating strategy. For example, Diminishing Marginal Utility should be taken into consideration when the system receives the feedback from the student. With diminishing marginal utility, the system can make the suggesting learning path more individualize. The second one is how the system can generate the navigating sentences without using the sentence templates. Furthermore, how the system navigates students to go to the next learning area with the direction instructions.

\section{Reference}

[1] Brown, J. S., Collins, A., \& Duguid, P. (1989). "Situated cognition and the cultural of learning," Educational Researcher, 18(1), pp.32-42

[2] Yuh-Shyan Chen, Tai-Chien Kao, Jang-Ping Sheu, and Chao-Yu Chiang (2002). “ A mobile scaffolding-aid-based bird-watching learning system," International Workshop on Wireless and Mobile Technologies in Education, pp.15-22

[3] Yuh-Shyan Chen, Tai-Chien Kao, Gwo-Jong Yu, and Jang-Ping Sheu (2004). "A mobile butterfly-watching learning system for supporting independent learning," The 2nd IEEE International
Workshop on Wireless and Mobile Technologies in Education, pp.11-18

[4] Collins, A. M. \& Quillian, M. R. (1969). "Retrieval time from semantic memory". Journal of Verbal Learning and Verbal Behavior, 8, 240-248.

[5] Freksa, C. (1992) "Using orientation information for qualitative spatial reasoning," In Frank, A. U., Campari, I., Formentini, U. (Eds.) Proceedings of the International Conference on Theories and Methods of Spatio-Temporal Reasoning in Geographic Space, Pisa, Italy, 1992, pp.162-178.

[6] T. Georgiev, E. Georgieva, and G. Trajkovski, (2006) "Transitioning from e-Learning to m-Learning: Present Issues and Future Challenges", Seventh ACIS International Conference on Software Engineering, Artificial Intelligence, Networking, and Parallel/Distributed Computing, 2006, pp. 349-353.

[7] Glasgow, J. I. and Papadias, D. (1992) "Computational imagery," Cognitive Science, 16, pp.355-394, 1992

[8] Kuo, R., Chang, M., Dong, D. X., Yang, K. Y., \& Heh, J. S. (2002). "Applying Knowledge Map to Intelligent Agents in Problem Solving Systems," In the Proceedings of the World Conference on Educational Multimedia, Hypermedia \& Telecommunications (ED-Media 2002), 24-29 June, 2002, Denver, Colorado, USA, 1053-1054.

[9] Lave, J., \& Wenger, E. (1991). Situated Learning: Legitimate peripheral participation. New York: Cambridge University Press.

[10] M.J. McAlister, and P.H. Xie, (2005) "Using a PDA for Mobile Learning", IEEE International Workshop on Wireless and Mobile Technologies in Education, 2005, pp. 282-284.

[11] Pullar, D. and Egenhofer, M. (1988) "Toward formal definitions of topological relations among spatial objects," Proceedings of the Third International Symposium on Spatial Data Handling, Sydney, Australia, 1988, pp.225-242

[12] Rumelhart, D. E. \& Norman, D. A. (1983). "Representation in memory," In R. C. Atkinson, R. J. Herrnstein, G. Lindzey, and R. D. Luce (Eds.), Handook of Experimental Psychology, Volume 2, Learning and Cognition, New York, NY: Wiley

[13] Vygotsky, L. S. (1994). "The problem of the environment," In R.v.d. Veer \& J. Valsiner (Eds.), The Vygotsky reader. Oxford, United Kingdom: Blackwell. 